\title{
Turismo. Tendencias Globales y Planificación Estratégica
}

\section{Tourism. Global trends and strategic planning}

\author{
Noemí Vega-Lugo ${ }^{a}$, Ivette Flores-Jiménez ${ }^{b}$ Liza Viviana Velazco-Álvarez ${ }^{c}$, Ruth Flores- \\ Jiménez ${ }^{b}$ José Sergio Rodríguez-Jiménez ${ }^{d}$
}

\section{Resumen}

El turismo es una de las actividades generadoras de recursos a nivel mundial, abordada desde diferentes puntos de vista por los estudiosos que se han ocupado de entenderla e impulsarla a lo largo de los años, su importancia como versatilidad ha permitido que se aborde desde diferentes puntos de vista, como lo es el comportamiento del turista, mercado turístico, espacio turístico, mantenimiento de la sostenibilidad y las nuevas tendencias del turismo, entre otros, temas que independientemente de su relevancia no es posible que se vean de manera aislada, es por ello que el autor que se cita en este trabajo trata de ver al turismo como un sistema en donde cada uno de sus componentes confluye para dar respuesta no solo a la exigencia de un mercado que demanda algo nuevo cada día sino de una comunidad receptora necesitada de recursos y de visibilidad hacia el ámbito nacional e internacional teniendo como punto de partida la planificación turística.

En esta nueva perspectiva es indispensable tener en cuenta la totalidad de los componentes de un sistema turístico representados por recursos naturales, culturales, económicos, políticos, sociales, cadenas productivas, prestadores de servicios, etc.

\section{Palabras clave:}

Turismo, Planificación, Sistema

\begin{abstract}
Tourism is one of the activities generating resources worldwide, approached from different points of view by scholars who have been trying to understand and promote it over the years, its importance as versatility has allowed it to be addressed from different points of sight, as it is the behavior of the tourist, tourist market, tourist space, maintenance of the sustainability and the new tendencies of the tourism, among others, subjects that independently of their relevance it is not possible that they are seen of isolated way, it is for it that the author cited in this paper tries to see tourism as a system where each of its components converges to respond not only to the demand of a market that demands something new every day but of a receiving community in need of resources and of visibility towards the national and international scope having as a starting point the tourist planning.

In this new perspective it is essential to take into account all the components of a tourism system represented by natural, cultural, economic, political, social, productive chains, service providers, etc.

\footnotetext{
a Autor de Correspondencia, Universidad Autónoma del Estado de Hidalgo, Instituto de Ciencias Económicas Administrativas. Email: noemivl@uaeh.edu.mx

${ }^{b}$ Profesor Investigador, Universidad Autónoma del Estado de Hidalgo, Escuela Superior de Tlahuelilpan. Email: Ivette Flores Jiménez: ivette_flores7@hotmail.com; Ruth Flores Jiménez: ruthfj_22@hotmail.com

c Docente del programa educativo en Turismo, del Instituto de Ciencias Económicas Administrativas, Universidad Autónoma del Estado de Hidalgo. Email: lizavv@uaeh.edu.mx

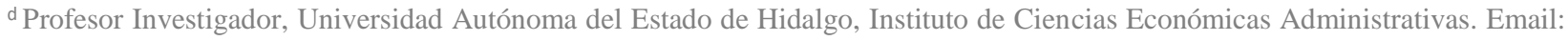
sergior@uaeh.edu.mx
} 
Keywords:

Tourism, Planning, System

\section{Reseña}

La obra mostrada por estos autores, quienes se han desempeñado como reconocidos docentes en diversas universidades de la Ciudad de Colombia impartiendo cátedras en las áreas de turismo y hotelería presentan en ocho capítulos un trabajo que resulta por demás interesante para quienes se dedican a la actividad turística, con un lenguaje claro y ameno parten en el primer capítulo "EI Turismo", de la conceptualización del mismo, los tipos de turistas, así como la evolución y las etapas por las que ha pasado esta noble actividad desde finales del siglo XVI hasta la etapa posmoderna, ubicando al lector en un contexto general para dar paso en el segundo capítulo a la descripción de la "Distribución espacial del turismo" que inicia con su definición, la configuración de las grandes áreas turísticas mundiales y concluyendo con los flujos turísticos mundiales determinantes para la planificación turística.

En un tercer capítulo describe “EI sistema turístico. Las unidades estadísticas y las variables del turismo desde la óptica de la demanda y la oferta", visualizando a este sector como un sistema conformado por elementos como: demanda, oferta, proceso de venta, producto turístico, atractivos, infraestructura, superestructura y patrimonio cada uno de ellos con una función específica y en donde la falla de uno automáticamente afectara a todos los demás.

El cuarto capítulo titulado "Teoría del espacio turístico y desarrollo del mercado turístico", sitúa al lector en entender el espacio turístico como "aquel espacio geográfico determinado donde se asienta la oferta y hacia el que fluye la demanda" describiendo así el espacio turístico natural y urbano, la jerarquización de las zonas, los tipos de destinos, el mercado, producto, estructura de la demanda, tipos de productos y servicios, oferta turística, para finalizar con aspectos de marketing.
Dentro del capítulo cinco cuyo título es "Mantenimiento de la sostenibilidad del turismo y las nuevas tendencias globales del turismo", definen la sustentabilidad, su importancia y su utilización como estrategia de desarrollo, dando pie al capítulo seis enmarcado como "La planificación turística" y en donde partiendo de los antecedentes se analizan los objetivos, actores y etapas básicas en el proceso de planificación turística. En el capítulo siete con la "Planificación estratégica del turismo" se describen detalladamente los criterios de esta planificación, sus componentes, así como las etapas, finalizando con el capítulo ocho donde refiere un "Modelo aplicativo de la planificación estratégica a los diferentes niveles de desarrollo turístico".

En conclusión la obra describe de manera clara la conceptualización del turismo denotando los componentes que lo caracterizan, con un sentido práctico que nos permite enfocarnos al desarrollo de elementos clave para contar con bases que permitan desarrollar la planeación del espacio turístico que va desde un enfoque tradicional hasta un enfoque estratégico.

Así mismo podemos afirmar que para el caso de profesores y alumnos que estudian este sector se convierte en una herramienta sumamente útil para asignaturas como introducción al turismo, desarrollo sustentable y medio ambiente, sustentabilidad turística y por supuesto planificación del espacio turístico, llevando a los actores antes mencionados no solo a tener conceptos claros sino a la aplicación de modelos que permiten una visualización integral del turismo. 


\section{Referencias}

\section{Reseña del Libro:}

Tendencias globales y planificación estratégica

Autor: Luis Hernando Jiménez Bulla y Wilson Giovanni

Jiménez Barbosa

Año: Reimpresión 2015

Editorial ECOE Ediciones

ISBN 978-958-648-852-5

1] Jiménez Bulla, L., \& Jiménez Barbosa , W. (2015). Turismo

Tendencias globales y planificación estratégica.

Bogota: ECOE Ediciones 